\title{
Violência física e fatores associados em participantes da Pesquisa Nacional de Saúde do Escolar (PeNSE)
}

\author{
Physical violence and associated factors in participants \\ of the National Student Health Survey (NSHS)
}

Juliana Souza Romeiro (https://orcid.org/0000-0003-1314-5789) ${ }^{1}$

Márcia Mara Corrêa (https://orcid.org/0000-0003-2042-0048) ${ }^{2}$

Rosalva Pazó (https://orcid.org/0000-0003-3469-1818) ${ }^{3}$

Franciele Marabotti Costa Leite (https://orcid.org/0000-0002-6171-6972) ${ }^{4}$

Nágela Valadão Cade (https://orcid.org/0000-0001-6073-504X) ${ }^{5}$
${ }^{1}$ Departamento de Saúde Coletiva, Universidade Federal do Espírito Santo (UFES). Av. Marechal Campos 1468, Bonfim. 29047-105 Vitória ES Brasil. julyromeiro@hotmail.com

${ }^{2}$ Programa de PósGraduação em Nutrição, Centro de Ciências da Saúde, UFES. Vitória ES Brasil.

${ }^{3}$ UFES. Vitória ES Brasil.

${ }^{4}$ Departamento de Enfermagem, Centro de Ciências da Saúde, UFES. Vitória ES Brasil.

${ }^{5}$ Programa de PósGraduação em Saúde Coletiva, Departamento de Enfermagem, UFES. Vitória ES Brasil.

\begin{abstract}
The objective of this article was to analyze the association between physical violen$c e$ in students with socioeconomic factors, family context, mental health, individual risky behaviors, and unsafe environment. Study with data from the National School Health Survey (NSHS) in 2015, with 9th graders. The outcome variable was the report of involvement in a physical struggle and the associated exposure variables used were related to socioeconomic and demographic conditions, family supervision and support, mental health, risky behaviors, and unsafe environment. Multivariate logistic regression with a hierarchical approach was used in the analyzes. The prevalence of involvement in a fight was higher in boys (30.2\%; CI 29.3-31.0) than in girls (16.7\%; CI 16.0-17.4). In both genders, there was a greater chance of involvement with physical violence when using drugs, missing classes, sedentary lifestyle, insomnia, loneliness and insecurity at school or in the community and, especially, when victimized by family aggression, OR 2.59 (CI 2.312.90) in boys and girls OR 2.42 (CI 2.17-2.71). There was a reduction in the chance of involvement in physical violence in boys because they were working and, in girls, when they study in a private school, having their problems and concerns welcomed by their parents or their participation in school activities.
\end{abstract}

Key words Violence, Adolescent, Adolescent behavior, School health
Resumo O objetivo deste artigo foi analisar a associação entre a violência física em escolares com fatores socioeconômicos, contexto familiar, saúde mental, comportamentos individuais de risco e ambiente inseguro. Estudo com os dados da Pesquisa Nacional de Saúde Escolar (PeNSE), 2015, com alunos do $9^{\circ}$ ano. A variável desfecho foi o relato de envolvimento em luta fisica e as variáveis de exposição associadas utilizadas foram relativas à condição socioeconômica e demográfica, supervisão e apoio familiar, saúde mental, comportamentos de risco e ambiente inseguro. Utilizou-se nas análises a regressão logística multivariada com abordagem hierarquizada. A prevalência de envolvimento em briga foi maior em meninos (30,2\%; IC 29,3-31,0) comparada às meninas (16,7\%; IC 16,0-17,4). Em ambos os sexos houve maior chance de envolvimento com violência física quando uso de drogas, falta às aulas, sedentarismo, insônia, solidão e insegurança na escola ou na comunidade e, principalmente, quando vítima de agressão familiar OR 2,59 (IC 2,31-2,90) em meninos e, em meninas OR 2,42 (IC 2,17-2,71). Houve redução da chance de envolvimento em violência física em meninos pelo fato de estarem trabalhando, em meninas, por estudarem em escola privada, e terem seus problemas e preocupações acolhidos pelos pais ou a participação destes nas atividades escolares.

Palavras-chave Violência, Adolescente, Comportamento do adolescente, Saúde escolar 


\section{Introdução}

$\mathrm{Na}$ adolescência é frequente a busca de desafios, independência e experimentações e a violência está dentre os comportamentos de risco mais abordados pela literatura e programas de saúde, nessa fase de vida ${ }^{1}$.

Segundo a OMS, os acidentes e violências representam o maior problema de saúde pública entre crianças e adolescentes em países em desenvolvimento ${ }^{2}$. Em 2009 foi acrescido ao Sistema de Informação de Agravos de Notificação do Ministério da Saúde, a notificação de violência doméstica extrafamiliar e comunitária, conforme o Estatuto da Criança e do Adolescente (ECA) e os dados mostraram que no ano de 2011, a faixa etária entre 15 e 19 anos foi a segunda na qual ocorreu mais atendimentos por violência no país ${ }^{3}$.

O Global Burden of Disease Study $y^{4}$ realizou avaliações anuais de doenças, sequelas e fatores de risco no período de 1990 a 2013 em 188 países e a violência interpessoal foi a quarta causa principal de morte em adolescentes de 15 a 19 anos de idade. $\mathrm{O}$ comportamento agressivo pode levar a diversos prejuízos para o adolescente, sua família e comunidade e dentre eles destacam-se os custos com atendimento médico, morbidades com sequelas permanentes, transtornos de aprendizagem, abstenção escolar, sentimentos de medo e óbito 5 . Ainda, os atos de violência interferem diretamente na qualidade de vida de toda uma sociedade ${ }^{6}$.

Quanto a prevalência em envolvimento em brigas e combate físico, estudo com 161.082 estudantes de 35 países da América do Norte e Europa, apontou valores que variaram de $37 \%$ a $69 \%$ em meninos e $17 \%$ a $32 \%$ em meninas? Já no Brasil as prevalências variam entre 16,2\% a $20,9 \% \%^{8,9}$.

Estudos realizados a partir das três últimas edições da Pesquisa Nacional de Saúde do Escolar (PeNSE) demonstraram a tendência temporal crescente de situações de violência, pois, aumentou as prevalências no período de 2009 a 2015 em todos os indicadores de violência avaliados ${ }^{10}$. Houve crescimento de 6,4\% para 12,8\% (variação média de 1,1 ) da quantidade de faltas as aulas por motivos de insegurança no trajeto escolar, as prevalências de faltas por motivos de violência no ambiente escolar aumentaram de 5,5 para 9,3, (variação média de 0,7 ), as brigas por arma de fogo variou de 4,0 para 5,6 (variação média de $0,3)$, enquanto briga com arma branca 6,1 para 8,2 (variação média de 0,4) e agressão física no ambiente familiar de 9,5 para 16,2 (variação média de 1,1). Foram encontrados nos estudos distintos padrões de crescimento da violência e de fatores de maior vulnerabilidade para tal, a exemplo estudar em instituições públicas e ser do sexo masculino ${ }^{10}$.

A interação de fatores nas esferas individual, relacional, comunitário e social podem influenciar no desenvolvimento da violência juvenil. Dentre alguns fatores gerais encontram-se a dificuldade para resolver os problemas pessoais, jovens que sofreram abusos ou agressões, ausência de supervisão e monitoramento parental, relações de namoro e de amizades, estar inserido em comunidade hostil e violenta, as oportunidades de educação, o suporte financeiro e as políticas públicas 5 .

Estudos internacionais de desenho transversal $^{11}$ e longitudinal ${ }^{12}$ demonstram a associação entre violência física na juventude e comportamentos de risco e estado emocional, e dentre eles destacam o uso de drogas ilícitas, álcool, fumo, iniciação sexual precoce, pobreza, vizinhança de elevada criminalidade, delinquência dos pares, baixa supervisão e apoio familiar, conflito parental, falta as aulas, baixo rendimento escolar, hiperatividade, bullying, comportamento antissocial, insônia, sintomas depressivos, ideação suicida e sedentarismo.

Diante do exposto, este estudo teve por objetivo em contexto nacional, analisar a associação entre a luta física em escolares com os fatores socioeconômicos, familiares, de saúde mental, comportamentos de risco e ambiente inseguro.

\section{Método}

Estudo de delineamento transversal que utilizou dados secundários da Pesquisa Nacional de Saúde do Escolar (PeNSE) de $2015^{13}$.

A PeNSE de 2015 é um estudo de base escolar sendo que a população analisada foi constituída por alunos do $9^{\circ}$ ano do ensino fundamental. A amostragem ocorreu por conglomerados, em dois estágios: as escolas como as unidades primárias e as turmas, como unidades secundárias, selecionadas aleatoriamente. De forma proporcional participaram alunos da rede de ensino público (municipais, estaduais e federais) e de ensino privado. Foram excluídos os participantes que não responderam as questões sobre o sexo e a idade, totalizando 102.072 questionários váli$\operatorname{dos}^{13}$. A coleta dos dados foi realizada em 2015, mediante questionário estruturado e autoaplicá- 
vel apresentado ao estudante, aplicados por técnicos do IBGE em smartphones ${ }^{13}$. A organização, planejamento e recursos para o desenvolvimento da pesquisa foi proveniente do Instituto Brasileiro de Geografia e Estatística (IBGE), em parceria com o Ministério da Saúde e apoio do Ministério da Educação ${ }^{13}$.

A variável dependente deste estudo constituiu o relato do envolvimento em briga do tipo luta física, seja como agressor ou vítima, pelo menos uma vez nos últimos doze meses. As variáveis de exposição avaliadas foram agrupadas em cinco blocos, sendo as condições socioeconômicas, demográfica e relativas à escola alocadas no bloco distal do modelo; as variáveis de comportamentos de risco, saúde mental, e supervisão/apoio familiar alocadas nos blocos intermediários, já as características de presença de ambiente inseguro, proximal ao desfecho.

Quanto ao bloco socioeconômico as variáveis foram idade, sexo, raçalcor, escolaridade materna, ter empregada mensalista e quantidade de banheiros com chuveiro no domicílio, ter trabalho remunerado ou não, número de moradores da casa e tipo de escola, se pública ou privada.

As variáveis de contexto e apoio familiar constituíram morar com o pai e/ou com a mãe, se nos últimos 30 dias os pais ou responsáveis sabiam o que o adolescente fazia no tempo livre, se compreendiam seus problemas e preocupações, se verificavam deveres ou tarefas de escola e se os escolares faziam refeições junto aos familiares.

Em relação a saúde mental as variáveis constituíram o sentimento de solidão, insônia nos últimos 12 meses e a existência de amigos próximos.

Quantos aos aspectos comportamentais foram analisados nos últimos 30 dias o consumo de drogas - álcool, fumo e drogas ilícitas -, prática de atividade física nos últimos sete dias e faltar as aulas pelo menos uma vez nos últimos 30 dias sem o conhecimento dos pais.

Sobre a atividade física foi utilizado o International Physical Activity Questionnaire (IPAQ), sendo considerados ativos adolescentes que praticaram um tempo igual ou superior a $30 \mathrm{minu}$ tos semanais ${ }^{14}$.

Com relação as variáveis sobre insegurança foram avaliadas a agressão familiar, a insegurança comunitária no trajeto casa escola e a insegurança na escola propriamente dita, nos últimos 30 dias.

A associação entre as variáveis de exposição e a violência física foi estimada pelo Teste do $\chi^{2}$ de Pearson, com nível de significância de $\mathrm{p} \leq 0,05$, as magnitudes foram medidas pelo Odds Ratio com IC95\% obtidos por meio de regressão logística multivariável, tendo a categoria "nunca brigou" como referência.

A seleção das variáveis de exposição que foram associadas a violência física com $\mathrm{p} \leq 0,20$, obtido em análises de regressão logística univariadas, foram incluídas na análise multivariável, sendo colocadas em blocos no modelo, onde cada um dos cinco blocos correspondeu a um nível hierárquico.

Para o modelo final foi usado o método de backward e permaneceram as variáveis associadas ao desfecho com $\mathrm{p} \leq 0,05$.

Para detectar a existência de multicolinearidade (dependência linear perfeita ou aproximada entre, pelo menos, duas variáveis explicativas), em cada um dos modelos multivariados ajustados, analisou-se a matriz de correlação entre os pares de coeficientes estimados. Nessas matrizes, correlações elevadas (geralmente superiores a 0,80-0,85) indicam um possível problema de colinearidade. Para cada modelo estimado, as respectivas matrizes foram analisadas, e não foram detectados sinais importantes de colinearidade.

Foi utilizado o pacote estatístico Stata (versão 13.1) e o comando $s v y$, adequado para análises de dados obtidos por plano amostral complexo.

Devido ao grande número de perdas de informação sobre escolaridade materna (em torno de $25 \%$ de respostas não obtidas), essa variável não foi considerada na análise multivariável.

A PeNSE foi aprovada pela Comissão Nacional de Ética em Pesquisas do Ministério da Saúde, (IBGE, 2016). Para participar da pesquisa os estudantes deveriam concordar com o Termo de Consentimento Livre e Esclarecido, localizado na primeira página do smartphone.

\section{Resultados}

$\mathrm{Na}$ amostra predominou jovens com idade entre 14 e 16 anos (78\%), ausência de empregada doméstica (90,5\%), com somente um banheiro com chuveiro $(61,7 \%)$, de escola pública $(85,6 \%)$, cujas mães tinham segundo grau completo (30,9\%). A agressão familiar esteve presente em $14,4 \%$ da população avaliada, já a agressão na escola ou no trajeto casa/escola foi constatada em $9,5 \%$ e em $11,4 \%$ dos escolares. A maioria nunca usou drogas lícitas (76\% para álcool e 93\% para fumo) ou ilícitas $(95,9 \%)$ e mais de $79 \%$ dos escolares foram classificados como sedentários. Moram com a mãe 89,9\% dos escolares avaliados 
e $43,8 \%$ relataram acolhimento dos pais quando na presença de problemas e preocupações. Realizam refeições com pais ou responsáveis $80,7 \%$ dos adolescentes, e $31,8 \%$ dos pais ou responsáveis verificam as lições ou tarefas de casa - dados não apresentados em tabela.

A prevalência de envolvimento em brigas, uma ou mais vezes nos últimos 12 meses (Tabela 1), foi de $23,3 \%$ (IC 22,6-23,8), maior em meninos 30,2\% (IC 29,3-31,0) comparado às meninas 16,7\% (IC 16,0-17,4).

$\mathrm{Na}$ análise bivariada, observa-se maior proporção de meninos com relato de violência física quando moravam sozinhos, residência com empregada e com dois ou mais banheiros, mãe com ensino superior, jovens que já trabalhavam, estudavam em escola privada, história de agressão familiar em casa e insegurança no entorno e na escola, comportamentos de consumo de drogas e falta as aulas, não morarem com a mãe ou com o pai e não perceberem apoio e supervisão dos pais. Em meninas, os resultados foram semelhantes, exceto relato de briga quando eram pretas ou de outras raças, ser filha de mãe analfabeta ou com fundamental incompleto, estudarem em escola pública, se sentirem sozinhas e não terem amigos (Tabela 2 e 3 ).

Foram oito os modelos de regressão logística tendo por base o modelo hierárquico. Em meninos houve ajuste para as variáveis idade, número de morador casa, ter empregada, banheiro, adolescente trabalhando e tipo de escola (modelo 1); exclusão da variável morador/casa (modelo 2); inserido no modelo 3 as variáveis referentes a supervisão/apoio familiar, sendo que todas mantiveram associação positiva conforme análise bivariada; inserido insônia, sentimento de solidão e quantidade de amigos próximos, relativas a saúde mental (modelo 4); excluída a variável sobre amigos (modelo 5); inserido o consumo de álcool, de droga e de fumo, se faltou as aulas e ativida- de física (modelo 6); excluído se mora com a mãe (modelo 7). No modelo final foram incluídas as variáveis agressão familiar, insegurança na escola e no trajeto casa/escola (Tabela 4).

Em meninas os modelos foram ajustados para as variáveis idade, raça/cor, número de morador casa, banheiro, adolescente trabalhando, se trabalho remunerado e tipo de escola (modelo 1); exclusão da variável morador casa (modelo 2); inserida as variáveis de supervisão/apoio familiar (modelo 3); excluída a variável se mora com a mãe (modelo 4) pois esta perdeu força de associação; inserido insônia, sentimento de solidão e quantidade de amigos próximos no modelo 5 , sendo excluída a variável sobre amigos no modelo 6; inseridos no modelo 7 as variáveis consumo de álcool, de droga e de fumo, se faltou as aulas e atividade física. Todas as variáveis incluídas no modelo mantiverem força de associação. No modelo final foram incluídas as variáveis agressão familiar, insegurança na escola e no trajeto casa/ escola (Tabela 5).

$\mathrm{Na}$ análise ajustada, nos dois sexos, aumentaram a chance de envolvimento em violência física nos últimos 12 meses quando consumo de drogas ilícitas, de álcool, de cigarro, ausência nas aulas sem permissão dos pais, sedentarismo, insônia, sentimento de solidão, insegurança na escola, no trajeto de casa/escola e agressão familiar. Ainda, nos meninos houve associação do envolvimento de brigas com estudar em escola privada, residência com dois ou mais banheiros, não morar com o pai (Tabelas 4 e 5).

Por outro lado, houve redução da chance de envolvimento em briga em meninos o fato de estarem trabalhando e, em meninas, $\mathrm{o}$ acolhimento dos problemas e preocupações pelos pais, conhecimento dos pais sobre as atividades da filha quando em tempo livre, e o fato de estudarem em escola privada (Tabelas 4 e 5).

Tabela 1. Prevalência de violência física em escolares brasileiros do $9^{\circ}$ ano do ensino fundamental. Brasil, PeNSE 2015.

\begin{tabular}{lrrr}
\hline \multicolumn{1}{c}{ Variável } & \multicolumn{1}{c}{ Todos \% (IC) } & Meninos \% (IC) & \multicolumn{1}{c}{ Meninas \% (IC) } \\
\hline Não & $76,7(76,1-77,3)$ & $69,8(68,9-70,6)$ & $83,3(82,5-83,9)$ \\
1 vez & $11,9(11,4-12,2)$ & $14,3(13,7-14,9)$ & $9,5(9,0-10,01)$ \\
2 vezes & $5,7(5,4-6,0)$ & $7,6(7,1-8,0)$ & $4,0(3,6-4,3)$ \\
3 vezes & $2,1(1,9-2,2)$ & $2,9(2,7-3,2)$ & $1,2(1,0-1,5)$ \\
$\geq 4$ vezes & $3,6(3,3-3,8)$ & $5,3(4,9-5,7)$ & $1,8(1,6-2,1)$ \\
\hline
\end{tabular}

Fonte: Pesquisa Nacional de Saúde Escolar, 2015. 
Tabela 2. Fatores associados a violência física segundo variáveis demográficas, socioeconômicas e relativas a escola entre adolescentes do $9^{\circ}$ ano escolar. Brasil, PeNSE 2015.

\begin{tabular}{|c|c|c|c|c|c|c|}
\hline \multirow{3}{*}{ Variáveis } & \multirow{2}{*}{\multicolumn{2}{|c|}{$\begin{array}{c}\text { Meninos } \\
\text { Violência Física } \\
\end{array}$}} & \multirow{3}{*}{ p-valor ${ }^{\star}$} & \multirow{2}{*}{\multicolumn{2}{|c|}{$\begin{array}{c}\text { Meninas } \\
\text { Violência Física } \\
\end{array}$}} & \multirow{3}{*}{ p-valor ${ }^{\star}$} \\
\hline & & & & & & \\
\hline & $\operatorname{Sim}(\%)$ & Não (\%) & & $\operatorname{Sim}(\%)$ & Não (\%) & \\
\hline Faixa etária (anos) & & & 0,129 & & & $<0,001$ \\
\hline $11-13$ & 28,6 & 71,4 & & 13,7 & 86,3 & \\
\hline $14-16$ & 30,5 & 69,5 & & 17,4 & 82,6 & \\
\hline$\geq 17$ & 31,4 & 68,6 & & 20,8 & 79,2 & \\
\hline Cor da pele & & & 0,264 & & & $<0,001$ \\
\hline Branca & 29,3 & 70,7 & & 15,0 & 85,0 & \\
\hline Preta & 30,7 & 69,1 & & 18,2 & 81,8 & \\
\hline Parda & 30,5 & 69,5 & & 17,4 & 82,6 & \\
\hline Outros & 31,9 & 68,1 & & 18,6 & 81,4 & \\
\hline No de morador/casa & & & $<0,001$ & & & 0,065 \\
\hline Mora sozinho & 73,6 & 26,4 & & 23,1 & 76,9 & \\
\hline 2 pessoas & 32,8 & 67,2 & & 19,1 & 80,9 & \\
\hline 3 pessoas & 28,8 & 71,2 & & 15,6 & 84,4 & \\
\hline$\geq 4$ pessoas & 30,3 & 60,7 & & 16,8 & 83,2 & \\
\hline Empregada doméstica & & & $<0,001$ & & & 0,896 \\
\hline Sim & 39,2 & 60,8 & & 16,9 & 83,1 & \\
\hline Não & 29,2 & 70,8 & & 16,7 & 83,3 & \\
\hline Banheiro/chuveiro & & & $<0,001$ & & & 0,033 \\
\hline Nenhum & 25,3 & 74,7 & & 13,6 & 86,4 & \\
\hline 1 & 28,0 & 72,0 & & 82,8 & 82,8 & \\
\hline$\geq 2$ & 34,5 & 65,5 & & 83,7 & 83,7 & \\
\hline Escolaridade da mãe & & & 0,002 & & & $<0,000$ \\
\hline Analfabeta & 28,4 & 71,6 & & 17,6 & 82,4 & \\
\hline Fund. Inc. & 29,1 & 70,9 & & 17,8 & 82,2 & \\
\hline Fund. Comp. & 32,7 & 67,3 & & 17,1 & 82,9 & \\
\hline $2^{0}$ grau Comp. & 31,5 & 68,5 & & 16,9 & 83,1 & \\
\hline Superior & 33,7 & 66,3 & & 13,1 & 86,9 & \\
\hline Adolescente trabalhando & & & $<0,001$ & & & $<0,001$ \\
\hline $\operatorname{Sim}$ & 41,8 & 58,2 & & 23,0 & 77,0 & \\
\hline Não & 27,7 & 72,3 & & 16,1 & 83,9 & \\
\hline Trabalho remunerado & & & $<0,001$ & & & $<0,001$ \\
\hline Nunca Trabalhou & 27,7 & 72,3 & & 16,1 & 83,9 & \\
\hline Sim & 41,8 & 58,2 & & 23,4 & 77,6 & \\
\hline Não & 40,8 & 59,2 & & 28,8 & 77,1 & \\
\hline Relativas à Escola & & & 0,617 & & & 0,160 \\
\hline \multicolumn{7}{|l|}{ Turno } \\
\hline Manhã/Tarde/Noite & 30,2 & 69,8 & & 16,7 & 83,3 & \\
\hline Integral & 32,3 & 67,7 & & 19,7 & 80,3 & \\
\hline Tipo & & & $<0,001$ & & & $<0,001$ \\
\hline Pública & 9,3 & 70,7 & & 17,5 & 82,5 & \\
\hline Privada & 35,5 & 64,5 & & 12,1 & 87,9 & \\
\hline
\end{tabular}

*Valor de $\mathrm{p}$ do teste de $\chi 2$ (qui-quadrado) entre as frequências de violência física nas categorias de análise.

Fonte: Pesquisa Nacional de Saúde Escolar, 2015.

\section{Discussão}

Os principais fatores associados a violência física em escolares do $9^{\circ}$ ano encontrados neste estudo constituíram a agressão familiar por mais de uma vez em 30 dias, entendendo-a como a convivência do jovem em um ambiente familiar inseguro e aqueles de natureza comportamental eviden- 
Tabela 3. Fatores associados a violência física segundo variáveis relativas aos comportamentos de risco, saúde mental, supervisão/apoio familiar e "ambiente seguro", entre adolescentes do $9^{\circ}$ ano escolar. Brasil, PeNSE 2015.

\begin{tabular}{|c|c|c|c|c|c|c|}
\hline \multirow{3}{*}{ Variáveis } & \multirow{2}{*}{\multicolumn{2}{|c|}{$\begin{array}{c}\text { Meninos } \\
\text { Violência Física }\end{array}$}} & \multirow{3}{*}{$\begin{array}{c}\text { p- } \\
\text { valor }^{*}\end{array}$} & \multirow{2}{*}{\multicolumn{2}{|c|}{\begin{tabular}{c|} 
Meninas \\
Violência Física
\end{tabular}}} & \multirow{3}{*}{$\begin{array}{c}\text { p- } \\
\text { valor }^{*}\end{array}$} \\
\hline & & & & & & \\
\hline & $\operatorname{Sim}(\%)$ & Não (\%) & & $\operatorname{Sim}(\%)$ & Não (\%) & \\
\hline \multicolumn{7}{|l|}{ Comportamentos de risco } \\
\hline Consumo de álcool nos últimos 30 dias & & & $<0,001$ & & & $<0,001$ \\
\hline Nunca usou & 19,5 & 80,5 & & 7,2 & 92,8 & \\
\hline Usa mas não bebeu nos 30 dias & 31,3 & 68,7 & & 18,3 & 81,7 & \\
\hline Bebeu pelo menos 1 vez em 30 dias & 50,1 & 49,9 & & 31,6 & 68,4 & \\
\hline Consumo de droga nos últimos 30 dias & & & $<0,001$ & & & $<0,001$ \\
\hline Nunca usou & 27,5 & 72,4 & & 14,3 & 85,7 & \\
\hline Consome mas não fez uso nos 30 dias & 50,9 & 49,1 & & 37,7 & 62,3 & \\
\hline Fez uso pelo menos 1 vez em 30 dias & 61,7 & 38,3 & & 50,8 & 49,2 & \\
\hline Consumo de fumo nos últimos 30 dias & & & $<0,001$ & & & $<0,001$ \\
\hline Nunca usou & 26,0 & 74,0 & & 12,5 & 87,5 & \\
\hline Usa mas não fumou nos 30 dias & 43,7 & 56,3 & & 31,8 & 68,2 & \\
\hline Fumou pelo menos 1 vez em 30 dias & 56,7 & 42,3 & & 49,0 & 51,0 & \\
\hline Atividade Física nos últimos 7 dias & & & $<0,001$ & & & $<0,001$ \\
\hline Sedentário & 28,4 & 71,6 & & 16,1 & 83,9 & \\
\hline Ativo & 34,8 & 65,2 & & 21,1 & 78,9 & \\
\hline $\begin{array}{l}\text { Faltou as aulas nos últimos } 30 \text { dias sem permissão } \\
\text { dos pais ou responsáveis }\end{array}$ & & & $<0,001$ & & & $<0,001$ \\
\hline Nenhum dia & 26,9 & 73,1 & & 13,8 & 86,2 & \\
\hline$\geq 1 \mathrm{dia}$ & 40,1 & 59,9 & & 27,3 & 72,7 & \\
\hline \multicolumn{7}{|l|}{ Saúde Mental } \\
\hline Insônia & & & $<0,001$ & & & $<0,001$ \\
\hline Não & 26,7 & 73,3 & & 11,8 & 88,2 & \\
\hline Sim & 39,8 & 60,2 & & 22,8 & 77,2 & \\
\hline Sentimento de solidão & & & $<0,001$ & & & $<0,001$ \\
\hline Não & 26,8 & 73,2 & & 12,3 & 87,7 & \\
\hline $\operatorname{Sim}$ & 37,6 & 62,4 & & 20,3 & 79,7 & \\
\hline Quantidade de amigos próximos & & & 0,954 & & & 0,004 \\
\hline Nenhum amigo & 30,1 & 69,9 & & 21,5 & 78,5 & \\
\hline$\geq 1$ amigo & 30,2 & 69,8 & & 16,5 & 83,5 & \\
\hline
\end{tabular}

ciados pelo consumo de drogas lícitas e ilícitas, sedentarismo e ausência nas aulas.

Pesquisas internacionais ${ }^{15,16}$ que se referem à luta física envolvendo escolares tem mostrado oscilação quanto as variáveis de exposição, entretanto, alguns comportamentos e perfis apresentados têm elevada frequência de associação. São eles: sexo masculino, consumo de drogas lícitas e ilícitas, faltar as aulas, morar em locais violentos, conflitos no sistema familiar, pouca supervisão parental e ter sintomas depressivos.

A agressão familiar pode sinalizar um ambiente hostil no domicílio, favorecendo a aprendizagem pelo jovem de modelos de comportamento agressivo desenvolvidos tanto no seio da família, como na sociedade. A vivência de crianças e adolescentes a situações de violência está relacionada a uma maior propensão a ter comportamentos que reproduzem agressividade nos relacionamentos atuais ou futuros ${ }^{17}$.

Estudos tanto nacionais, como internacionais corroboram com a relação entre agressão no ambiente intrafamiliar e comportamentos violentos na infância e adolescência. Em Fortaleza-CE foi identificado que apanhar em casa aumentou em $13 \%$ o envolvimento do jovem em violência interpessoal como agressor ${ }^{18}$. Em São Gonçalo-RJ, inquérito evidenciou que alunos que referiram violência psicológica, sexual ou física severa por algum familiar ou pessoas de relacionamentos 
Tabela 3. Fatores associados a violência física segundo variáveis relativas aos comportamentos de risco, saúde mental, supervisão/apoio familiar e "ambiente seguro", entre adolescentes do $9^{\circ}$ ano escolar. Brasil, PeNSE 2015.

\begin{tabular}{|c|c|c|c|c|c|c|}
\hline \multirow{3}{*}{ Variáveis } & \multirow{2}{*}{\multicolumn{2}{|c|}{$\begin{array}{c}\text { Meninos } \\
\text { Violência Física }\end{array}$}} & \multirow{3}{*}{$\begin{array}{c}\text { p- } \\
\text { valor }^{*}\end{array}$} & \multirow{2}{*}{\multicolumn{2}{|c|}{$\begin{array}{c}\text { Meninas } \\
\text { Violência Física }\end{array}$}} & \multirow{3}{*}{$\begin{array}{c}\text { p- } \\
\text { valor }^{*}\end{array}$} \\
\hline & & & & & & \\
\hline & $\operatorname{Sim}(\%)$ & Não (\%) & & $\operatorname{Sim}(\%)$ & Não (\%) & \\
\hline \multicolumn{7}{|l|}{ Supervisão e Apoio Familiar } \\
\hline Mora com a mãe & & & $<0,000$ & & & $<0,000$ \\
\hline Sim & 29,7 & 70,3 & & 16,3 & 83,7 & \\
\hline Não & 35,2 & 64,8 & & 20,8 & 79,2 & \\
\hline Mora com o pai & & & $<0,000$ & & & $<0,000$ \\
\hline Sim & 28,4 & 71,6 & & 15,1 & 84,9 & \\
\hline Não & 33,9 & 66,1 & & 19,4 & 80,6 & \\
\hline Realizar refeições com pais ou responsáveis & & & $<0,000$ & & & $<0,000$ \\
\hline Sim & 29,2 & 70,8 & & 14,9 & 85,1 & \\
\hline Não & 36,0 & 64,0 & & 23,1 & 76,9 & \\
\hline $\begin{array}{l}\text { Problemas e preocupações acolhidos por pais ou } \\
\text { responsáveis }\end{array}$ & & & $<0,000$ & & & $<0,000$ \\
\hline $\operatorname{Sim}$ & 28,2 & 71,8 & & 13,7 & 86,3 & \\
\hline Não & 35,2 & 64,8 & & 22,0 & 78,0 & \\
\hline $\begin{array}{l}\text { Pais ou responsáveis sabiam o que estava fazendo no } \\
\text { tempo livre }\end{array}$ & & & $<0,000$ & & & $<0,000$ \\
\hline $\operatorname{Sim}$ & 28,3 & 71,7 & & 14,8 & 85,2 & \\
\hline Não & 37,2 & 62,8 & & 25,8 & 74,2 & \\
\hline \multicolumn{7}{|l|}{ Ambiente Seguro } \\
\hline Agressão Familiar nos últimos 30 dias & & & $<0,001$ & & & $<0,001$ \\
\hline Nenhuma vez & 25,8 & 74,2 & & 13,0 & 87,0 & \\
\hline$\geq 1 \mathrm{vez}$ & 57,5 & 42,5 & & 37,7 & 62,3 & \\
\hline Insegurança na escola nos últimos 30 dias & & & $<0,001$ & & & $<0,001$ \\
\hline Nenhum dia & 27,5 & 72,5 & & 15,2 & 84,8 & \\
\hline$\geq 1$ dia & 53,9 & 46,1 & & 32,5 & 67,5 & \\
\hline $\begin{array}{l}\text { Insegurança no trajeto casa/escola nos últimos } 30 \\
\text { dias }\end{array}$ & & & $<0,001$ & & & $<0,001$ \\
\hline Nenhum dia & 27,7 & 72,3 & & 15,0 & 85,0 & \\
\hline$\geq 1$ dia & 49,5 & 50,5 & & 30,3 & 69,7 & \\
\hline Pais ou responsáveis verificavam lições ou tarefas & & & $<0,000$ & & & $<0,000$ \\
\hline Sim & 28,0 & 72,0 & & 13,8 & 86,2 & \\
\hline Não & 33,5 & 66,5 & & 19,9 & 80,1 & \\
\hline
\end{tabular}

*Valor de $\mathrm{p}$ do teste de $\chi 2$ (qui-quadrado) entre as frequências de violência física nas categorias de análise.

significativos aumentou mais de três vezes a chance de se envolverem em violência na comunidade e no ambiente escolar, em relação aos alunos que não relataram violência doméstica ${ }^{19}$. Em Minessota, Estados Unidos (EUA), os resultados foram semelhantes mesmo quando avaliado abuso físico ou sexual por um membro familiar ou não, ou simplesmente presenciar abuso físico na família ${ }^{20}$.

Diversas formas de manifestações da violência podem contribuir para a sensação de insegurança nas escolas, são exemplos o bullying, uso de armas de fogo ou arma branca, luta física, roubos, danos ao patrimônio, consumo de drogas e a violência no entorno ou bairro em que a escola está inserida ${ }^{10,21}$. A exposição a violência na escola e comunidade podem comprometer o desempenho acadêmico, a frequência escolar, causar danos físicos e emocionais ${ }^{22}$ e a sensação de insegurança é comumente verificada com maior frequência em escolas públicas, por motivos de localização ou aspectos relacionados a organização escolar ${ }^{10}$. 
Tabela 4. Odds Ratios bruto e ajustado no modelo final de violência física segundo categorias de análise entre adolescentes do sexo masculino do $9^{\circ}$ ano escolar. Brasil, PeNSE 2015.

\begin{tabular}{|c|c|c|c|c|}
\hline \multirow{3}{*}{ Variáveis } & \multicolumn{4}{|c|}{ Meninos } \\
\hline & \multirow{2}{*}{$\begin{array}{c}\text { Análise Bruta } \\
\text { Odds Ratio } \\
\text { (IC95\%) }\end{array}$} & \multirow[b]{2}{*}{ p-valor* } & \multirow{2}{*}{$\begin{array}{c}\text { Análise Ajustada } \\
\text { Odds Ratio } \\
\text { (IC95\%) }\end{array}$} & \multirow[b]{2}{*}{ p-valor ${ }^{\star}$} \\
\hline & & & & \\
\hline Banheiro/chuveiro & & $<0,001$ & & $<0,001$ \\
\hline Nenhum & 1,00 & & 1,00 & \\
\hline 1 & $1,14(0,94-1,38)$ & & $1,23(1,02-1,50)$ & \\
\hline$\geq 2$ & $1,55(1,28-1,87)$ & & $1,56(1,29-1,90)$ & \\
\hline Adolescente trabalhando & & $<0,001$ & & $<0,001$ \\
\hline Sim & 1,00 & & 1,00 & \\
\hline Não & $0,53(0,48-0,58)$ & & $0,76(0,69-0,85)$ & \\
\hline Relativas à Escola - Tipo & & $<0,001$ & & $<0,001$ \\
\hline Pública & 1,00 & & 1,00 & \\
\hline Privada & $1,32(1,18-1,48)$ & & $1,52(1,35-1,72)$ & \\
\hline Mora com o pai & & $<0,001$ & & 0,004 \\
\hline Sim & 1,00 & & 1,00 & \\
\hline Não & $1,29(1,20-1,39)$ & & $1,12(1,04-1,23)$ & \\
\hline Insônia & & $<0,001$ & & $<0,001$ \\
\hline Sim & $1,80(1,65-1,97)$ & & $1,27(1,15-1,35)$ & \\
\hline Não & 1,00 & & 1,00 & \\
\hline Sentimento de solidão & & $<0,001$ & & $<0,001$ \\
\hline Sim & $1,64(1,52-1,76)$ & & $1,24(1,04-1,38)$ & \\
\hline Não & 1,00 & & 1,00 & \\
\hline Consumo de álcool nos últimos 30 dias & & $<0,001$ & & $<0,001$ \\
\hline Nunca usou & 1,00 & & 1,00 & \\
\hline Usa mas não bebeu nos 30 dias & $1,87(1,71-2,06)$ & & $1,57(1,43-1,72)$ & \\
\hline Bebeu pelo menos 1 x em 30 dias & $1,79(1,32-2,44)$ & & $2,49(2,23-2,78)$ & \\
\hline Consumo de droga nos últimos 30 dias & & $<0,001$ & & $<0,001$ \\
\hline Nunca usou & 1,00 & & 1,00 & \\
\hline Consome mas não fez uso nos 30 dias & $2,72(2,29-3,23)$ & & $1,39(1,14-1,70)$ & \\
\hline Fez uso pelo menos 1 vez em 30 dias & $4,24(3,60-4,98)$ & & $1,55(1,26-1,90)$ & \\
\hline Consumo de fumo nos últimos 30 dias & & $<0,001$ & & $<0,001$ \\
\hline Nunca usou & 1,00 & & 1,00 & \\
\hline Usa mas não fumou nos 30 dias & $2,21(1,99-2,45)$ & & $1,32(1,17-1,49)$ & \\
\hline Fumou pelo menos $1 \mathrm{vez}$ em 30 dias & $3,88(3,35-4,48)$ & & $1,43(1,16-1,72)$ & \\
\hline Atividade Física & & $<0,001$ & & $<0,001$ \\
\hline Ativo & 1,00 & & 1,00 & \\
\hline Sedentário & $1,34(1,24-1,46)$ & & $1,35(1,24-1,48)$ & \\
\hline $\begin{array}{l}\text { Faltou as aulas nos últimos } 30 \text { dias sem permissão } \\
\text { dos pais ou responsáveis }\end{array}$ & & $<0,001$ & & $<0,001$ \\
\hline Nenhum dia & 1,00 & & 1,00 & \\
\hline$\geq 1$ dia & $1,81(1,65-1,98)$ & & $1,31(1,18-1,46)$ & \\
\hline Agressão Familiar nos últimos 30 dias & & $<0,001$ & & $<0,001$ \\
\hline Nenhum vez & 1,00 & & 1,00 & \\
\hline$\geq 1 \mathrm{vez}$ & $3,89(3,52-4,29)$ & & $2,59(2,31-2,90)$ & \\
\hline $\begin{array}{l}\text { Insegurança no trajeto casa/escola nos últimos } 30 \\
\text { dias }\end{array}$ & & $<0,001$ & & 0,003 \\
\hline Nenhum dia & 1,00 & & 1,00 & \\
\hline$\geq 1$ dia & $3,08(2,72-3,48))$ & & $1,24(1,07-1,43)$ & \\
\hline Insegurança na escola nos últimos 30 dias & & $<0,000$ & & $<0,000$ \\
\hline Nenhum dia & 1,00 & & 1,00 & \\
\hline$\geq 1$ dia & $2,56(2,31-2,83)$ & & $1,68(1,41-2,00)$ & \\
\hline
\end{tabular}


Tabela 5. Odds Ratios bruto e ajustado no modelo final de violência física segundo categorias de análise entre meninas do $9^{\circ}$ ano escolar. Brasil, PeNSE, 2015.

\begin{tabular}{|c|c|c|c|c|}
\hline \multirow{3}{*}{ Variáveis } & \multicolumn{4}{|c|}{ Meninas } \\
\hline & \multirow{2}{*}{$\begin{array}{c}\text { Análise Bruta } \\
\text { Odds Ratio } \\
\text { IC95\% }\end{array}$} & \multirow[b]{2}{*}{$\begin{array}{c}\text { p- } \\
\text { valor }^{*}\end{array}$} & \multirow{2}{*}{$\begin{array}{c}\text { Análise Ajustada } \\
\text { Odds Ratio } \\
\text { IC95\% }\end{array}$} & \multirow{2}{*}{$\begin{array}{c}\text { p- } \\
\text { valor }^{*}\end{array}$} \\
\hline & & & & \\
\hline Tipo de Escola & & $<0,001$ & & $<0,001$ \\
\hline Pública & 1,00 & & 1,00 & \\
\hline Privada & $0,64(0,57-0,73)$ & & $0,78(0,69-0,89)$ & \\
\hline $\begin{array}{l}\text { Problemas e preocupações acolhidos por pais ou } \\
\text { responsáveis }\end{array}$ & & $<0,001$ & & 0,015 \\
\hline Sim & $0,56(0,50-1,61)$ & & $0,89(0,71-0,93)$ & \\
\hline Não & 1,00 & & 1,00 & \\
\hline $\begin{array}{l}\text { Pais ou responsáveis sabiam o que estava fazendo no } \\
\text { tempo livre }\end{array}$ & & $<0,001$ & & 0,006 \\
\hline Sim & $0,49(0,44-0,56)$ & & $0,81(0,71-0,93)$ & \\
\hline Não & 1,00 & & 1,00 & \\
\hline Insônia & & $<0,001$ & & $<0,001$ \\
\hline Sim & $2,21(2,02-2,42)$ & & $1,42(1,27-1,59)$ & \\
\hline Não & 1,00 & & 1,00 & \\
\hline Sentimento de solidão & & $<0,001$ & & 0,015 \\
\hline Sim & $1,81(1,64-2,00)$ & & $1,13(1,01-1,26)$ & \\
\hline Não & 1,00 & & 1,00 & \\
\hline Consumo de álcool nos últimos 30 dias & & $<0,001$ & & $<0,001$ \\
\hline Nunca usou & 1,00 & & 1,00 & \\
\hline Usa mas não bebeu nos 30 dias & $2,87(2,55-3,22)$ & & $2,08(1,83-2,35)$ & \\
\hline Bebeu pelo menos $1 \mathrm{x}$ em 30 dias & $5,92(5,27-6,66)$ & & $2,82(2,44-3,26)$ & \\
\hline Consumo de droga nos últimos 30 dias & & $<0,001$ & & $<0,001$ \\
\hline Nunca usou & 1,00 & & 1,00 & \\
\hline Consome mas não fez uso nos 30 dias & $3,61(3,04-4,29)$ & & $1,38(1,12-1,70)$ & \\
\hline Fez uso pelo menos 1 vez em 30 dias & $6,16(5,00-7,60)$ & & $1,70(1,32-2,19)$ & \\
\hline Consumo de fumo nos últimos 30 dias & & $<0,001$ & & $<0,001$ \\
\hline Nunca usou & 1,00 & & 1,00 & \\
\hline Usa mas não fumou nos 30 dias & $3,27(2,90-3,69)$ & & $1,62(1,40-1,87)$ & \\
\hline Fumou pelo menos 1 vez em 30 dias & $6,74(5,73-7,93)$ & & $2,15(1,75-2,65)$ & \\
\hline Atividade Física & & $<0,001$ & & $<0,001$ \\
\hline Ativo & 1,00 & & 1,00 & \\
\hline Sedentário & $1,39(1,25-1,55)$ & & $1,33(1,17-1,50)$ & \\
\hline $\begin{array}{l}\text { Faltou as aulas nos últimos } 30 \text { dias sem permissão } \\
\text { dos pais ou responsáveis }\end{array}$ & & $<0,001$ & & $<0,001$ \\
\hline Nenhum dia & 1,00 & & 1,00 & \\
\hline$\geq 1$ dia & $2,33(2,09-2,60)$ & & $1,31(1,16-1,49)$ & \\
\hline Agressão familiar nos últimos 30 dias & & $<0,001$ & & $<0,001$ \\
\hline Nenhuma vez & 1,00 & & 1,00 & \\
\hline$\geq 1 \mathrm{vez}$ & $4,04(3,65-4,47)$ & & $2,42(2,17-2,71)$ & \\
\hline $\begin{array}{l}\text { Insegurança no trajeto casa/escola nos últimos } 30 \\
\text { dias }\end{array}$ & & $<0,001$ & & $<0,001$ \\
\hline Nenhum dia & 1,00 & & 1,00 & \\
\hline$\geq 1$ dia & $2,46(2,17-2,80)$ & & $1,48(1,27-1,72)$ & \\
\hline Insegurança na escola nos últimos 30 dias & & $<0,000$ & & 0,005 \\
\hline Nenhum dia & 1,00 & & 1,00 & \\
\hline$\geq 1$ dia & $2,69(2,36-3,06)$ & & $1,29(1,09-1,54)$ & \\
\hline
\end{tabular}


O consumo de álcool e outras drogas têm elevada prevalência entre os adolescentes e apesar de ter havido redução do consumo de cigarros nessa fase da vida, nos últimos anos tem elevado o consumo do álcool e drogas ilícitas o que tem mostrado associação com os comportamentos violentos ${ }^{23}$.

Inquérito nos $\mathrm{EUA}^{24}$ mostrou que os alunos que não fizeram uso de maconha e álcool foram os que menos se envolveram em brigas (77,3\%). Já na Tailândia, em estudantes que bebiam houve aumento de $200 \%$ de envolvimento em brigas sem lesões e $40 \%$ em brigas com lesões graves ${ }^{25}$.

No Brasil avaliaram a violência urbana, seja como agressor ou como vítima em pessoas entre 15 e 64 anos e as prevalências foram maiores em usuários de cocaína 19,7\% e em pessoas que consumiam frequentemente bebida alcoólica $18,1 \%{ }^{26}$.

Tem sido investigado os preditores do consumo de álcool, drogas ilícitas e a violência física na juventude, todavia tem havido dificuldade para estabelecer relações causais entre esses comportamentos, devido à complexidade para definir a ordem desses comportamentos ${ }^{26,27}$.

O comprometimento da saúde mental com a presença de insônia e sentimento de solidão, também aumentaram a chance da violência interpessoal, o que pode ser um indicativo de distúrbio de ansiedade, de depressão ou um Transtorno Mental Comum. Pesquisas evidenciam que adolescentes com sintomas depressivos, sentimentos de solidão e de ansiedade têm maior probabilidade para o envolvimento em comportamentos agressivos seja como vítimas ou como agressores $^{28-30}$.

Uma pesquisa transversal, que utilizou dados de base escolar na Arábia Saudita investigou por meio do auto relato a relação entre a violência física no último ano e a saúde mental de 9.073 estudantes. Foi encontrado associação positiva entre sintomas de depressão e ansiedade com a violência interpessoal. Escolares que se envolveram em briga tiveram 1,7 vezes mais chance de ter sintomas depressivos, enquanto escolares que relataram sinais de ansiedade tiveram 1,48 vezes mais chance de brigas e 1,84 mais chance de apresentar comprometimento da saúde mental, quando comparados a alunos que não referiram luta física ${ }^{30}$.

No Brasil, dados da Pesquisa Nacional de Saúde do Escolar (PeNSE) de 2015 evidenciou associação entre os sintomas depressivos, o sentimento de solidão e a insônia com o consumo de drogas lícitas e ilícitas, demonstrando a tendên- cia para o agrupamento de comportamentos de risco na adolescência ${ }^{31}$.

Neste estudo, em meninos, a ausência da figura paterna no domicílio foi associada à violência física, o que pode sinalizar a falta de um modelo de família nuclear. Estudo em São Paulo evidenciou uma prevalência maior de ações que envolvem criminalidade e violência, comportamento sexual de risco e consumo de drogas e álcool em jovens que não residem com nenhum dos pais e, em seguida, os jovens que moravam apenas com um dos pais justificando a importância da estrutura familiar para a prevenção de comportamentos de risco na juventude ${ }^{32}$.

Já nas meninas, aquelas que perceberam receber apoio familiar no dia a dia se envolveram menos em luta física, o que vai ao encontro de outros trabalhos investigativos que enfatizam a importância do suporte e apoio familiar como um fator que protege o jovem quanto aos comportamentos de risco ${ }^{29,33}$.

Uma melhor condição econômica em meninos evidenciada pelas variáveis proxy estudar em escola privada e ter dois ou mais banheiros em casa, aumentou a possibilidade de envolvimento em brigas. $\mathrm{Na}$ análise bivariada observou-se uma prevalência maior de mães com ensino superior em meninos que relataram briga, e esse contexto indicativo de classe social mais elevada tem sido controverso nos estudos que avaliam qualquer tipo de violência em jovens. Destaca-se que em estudo realizado com dados da PeNSE 2009, que não foi observada diferença quanto ao envolvimento em brigas entre as escolas públicas e as privadas ${ }^{21}$.

Marcadores de classe social mais baixa tem sido relacionado com violência em adolescente, conforme demonstrado por Kipping et al..$^{34}$, que observou em um estudo de coorte no Reino Unido que o status socioeconômico analisado mediante escolaridade materna, renda familiar, e classe social dos pais, que o nível social mais baixo se mostrou associado ao comportamento violento e diversas outras condutas prejudicais a saúde do adolescente ${ }^{34}$.

O espaço escolar privado em geral oferece mais segurança, controle disciplinar e melhor estrutura para a aprendizagem e desenvolvimento dos joven $s^{35}$. Embora pareça contraditório, alguns estudos também mostraram maior prevalência de comportamentos de risco em alunos da rede de ensino particular com relação a escolas públicas, e as condutas de risco mais observadas foram dirigir de forma imprudente, envolvimento em brigas, consumo de álcool, cigarro e drogas ilícitas ${ }^{36,37}$. 
Albuhairanl et al. ${ }^{30}$, encontraram associação entre a escolaridade materna mais elevada e o relato de estudantes de envolvimento em brigas no último ano, na Arábia Saudita ${ }^{30}$. Os pesquisadores relatam a natureza incomum desses achados e sugerem que poderia ser devido ao fato de a quantidade de mães com nível mais elevado de escolaridade representarem uma pequena parte da amostra (23,3\%).

Sabe-se que a evasão e o abandono escolar são sobremaneira maiores em alunos da rede pública, principalmente ao final do ensino fundamental e no ensino médio, podendo-se inferir que parte dos alunos que se envolvem em brigas possam estar fora da escola pública, levando a uma amostra deste estudo com perda desproporcional de escolares no que diz respeito a variável luta física ${ }^{38}$.

No que se refere ao trabalho dos adolescentes foi possível identificar essa variável neste estudo como fator de proteção para o envolvimento de violência interpessoal em meninos, o que é corroborado por outro estudo nacional ${ }^{39}$.

Foi também verificado o aumento de comportamentos de violência física entre os escolares que faltaram as aulas sem permissão dos pais em seis países do Pacífico Ocidental e entre os estudantes que faltaram as aulas sem a permissão dos pais tiveram maior propensão para brigas, $\mathrm{OR}=1,72(1,51-1,95)$ em relação aos estudantes que não faltaram as aulas ${ }^{11}$. Estudantes que faltam as aulas têm maior propensão a terem outros comportamentos de risco para a saúde como uso de álcool e drogas, a simultaneidade de fatores de risco a saúde aumenta a chance de envolvimento em situações de violência ${ }^{8,11}$.

As pesquisas sobre luta física envolvendo escolares oscilam de forma ampla com relação as variáveis associadas a esse desfecho. Entretanto, alguns comportamentos e perfis apresentados na literatura têm elevada frequência de associação com a violência física. São eles: sexo masculino, consumo de álcool, cigarro e drogas, faltar as aulas, morar em locais com elevados índice de violência, ter sintomas depressivos, conflitos no sistema familiar e pouca supervisão parental ${ }^{11,15,30,40}$. Esses achados se assemelham as variáveis encontradas nesse estudo que apresentaram associação com a violência física.

A literatura tem demonstrado que fatores contextuais e individuais influenciam no comportamento agressivo, e as intervenções para conter a violência devem estar embasadas em evidências científicas que sinalizam a violência em jovens que também estão sob a supervisão da escola, espaço esse com grande potencial para observação e intervenção com vistas a redução dos conflitos. Compreender as vulnerabilidades no qual os adolescentes estão inseridos é essencial para formular estratégias para a promoção da saúde de acordo com as suas necessidades e vivências de modo a proporcionar subsídios para o direcionamento das políticas públicas, promover a melhoria dos indicadores de saúde e a prevenção de comportamentos de risco nessa fase.

Como limitações do estudo além do desenho transversal que não se propõe a inferência causal, há a possiblidade maior de viés de informação, considerando a faixa etária envolvida, principalmente ao responderem perguntas subjetivas ou que precise de conhecimento e retenção deste, como a escolaridade da mãe.

A PeNSE só avalia as crianças que estão matriculadas e presentes na escola, mas o abandono escolar em jovens é elevado no país, principalmente daqueles de escola pública e com idade mais avançada, ao final do ensino fundamental ou no ensino médio.

Apesar das limitações, este estudo têm uma boa representatividade dos adolescentes do Brasil e traz contribuições para compreensão dos fatores associados ao envolvimento em brigas de escolares

Os principais fatores associados a violência, tipo luta física, relatada por meninos e meninas, estudantes do $9^{\circ}$ ano, são devido ao sentimento de insegurança principalmente a intrafamiliar decorrente da agressão familiar, mas também aquela percebida na comunidade e na escola e os fatores de natureza comportamental como o consumo de drogas lícitas (fumo e álcool), drogas ilícitas, sedentarismo e faltar às aulas. $\mathrm{O}$ comprometimento da saúde mental também esteve associado ao envolvimento em brigas, e em meninos, a ausência da figura paterna no domicílio.

Os fatores relacionados ao envolvimento em brigas em meninas e meninos foram de natureza semelhantes, mas as condições que as reduzem em meninos foi estar trabalhando e em meninas a percepção de receber apoio dos pais e estar em escola privada.

Múltiplos fatores estão associados ao envolvimento em violência física em adolescentes, seja como vítima ou agressor, evidenciando que um estilo de vida pouco saudável dos jovens e maior vulnerabilidade social, o que reforça a necessidade de programas que contemplem a complexidade e a coexistência das causas relacionadas. 


\section{Colaboradores}

JS Romeiro participou da idealização do artigo, trabalhou na interpretação dos dados e na redação do artigo. MM Corrêa trabalhou na análise e interpretação dos dados, realizou a revisão crítica e aprovou a versão final do manuscrito. R Pazó e FMC Leite colaboraram na revisão crítica e aprovaram a versão final. NV Cade participou da idealização do artigo, contribuiu na análise e interpretação dos dados, na revisão crítica e aprovou a versão final.

\section{Referências}

1. Cruzeiro ALS, Silva RA, Horta BL, Souza LDM, Faria AD, Pinheiro RT, Silveira IO, Ferreira CD. Prevalência e fatores associados ao transtorno da conduta entre adolescentes: um estudo de base populacional. Cad Saúde Pública 2008; 24(9):2013-2020.

2. World Health Organization (WHO). World Health Organization Improving Health trough schools: national and international strategies. Geneva: WHO; 1999.

3. Waiselfisz JJ. Mapa da Violência 2013: Homicídios e Juventude no Brasil. Brasília: Secretaria-Geral da Presidência da República, Secretaria Nacional de Juventude, Secretaria de Políticas de Promoção da Igualdade Racial; 2013.

4. Mokdad AH, Forouzanfar MH, Daoud, F, Mokdad, AA, Bchearaoui CE, Moradi-Lakeh M, Kyu HH, Barber RM, Wagner J, Cercy K, Kravitz H, Coggeshall M, Chew A, O'Rourke KF, Steiner C, Tuffaha M, Charara $\mathrm{R}, \mathrm{Al}$-Ghamdi EA, Adi Y, Afifi RA, Alahmadi $\mathrm{H}$, AlBuhairan F, Allen N, AlMazroa M, Al-Nehmi AA, AlRayess Z, Arora M, Azzopardi P, Barroso C, Basulaiman M, Bhutta ZA, Bonell C, Breinbauer C, Degenhardt L, Denno D, Fang J, Fatusi A, Feigl AB, Kakuma R, Karam N, Kennedy E, Khoja TAM, Maalouf F, Obermeyer CM, Mattoo A, McGovern T, Memish ZA, Mensah GA, Patel V, Petroni S, Reavley N, Zertuche DR, Saeedi M, Santelli J, Sawyer SM, Ssewamala F, Taiwo K, Tantawy M, Viner RM, Waldfogel J, Zuñiga MP, Naghavi M, Wang H, Vos T, Lopez AD, Al Rabeeah AA, Patton GC, Murray CJL. Global burden of diseases, injuries, and risk factors for young people's health during 1990-2013: A systematic analysis for the global burden of disease study 2013. Lancet 2016; 387:2383-2401.

5. Ferdon DC, Simon TR. Preventing Youth Violence: Opportunities for Action. Atlanta: National Center for Injury Prevention and Control, CDC; 2014.

6. Organização Pan-Americana de Saúde (OPAS). Relatório mundial de Violência e Saúde. Washington: OPAS; 2003.

7. Pickett W, Craig W, Harel Y, Cunningham J, Simpsom K, Molcho M, Mazur J, Dostaler S, Overpeck $\mathrm{MD}$, Currie CE, HBSC Violence and Injuries Writing Group. Cross-national study of fighting and weapon carrying as determinants of adolescent injury. Pediatrics 2005; 116(6):855-863.

8. Leão AS, Moura SNM, Gonçalves ECA, Silva DAS, Silva RJDS, Thomazzi SM. Simultaneous Health Risk Behaviors in Adolescents Associated with Higher Economic Class in the Northeast of Brazil. ScientificWorldJournal 2017; 3587567.

9. Malta DC, Mascarenhas, MDM, Dias, AR, Prado, RR, Lima CM, Silva MMA, Silva Júnior JB. Tendências para situações de violência vivenciadas por estudantes nas capitais brasileiras e no Distrito Federal: resultados da Pesquisa Nacional de Saúde do Escola (PeNSE 2012). Rev Bras Epidemiol 2014; 17(Supl. 1):158-171.

10. Pinto IV, Barufaldi LA, Campos MO, Malta DC, Rayone MCVS, Freitas MG, Lima CM, Andreazzi MAR. Tendências de situações de violência vivenciadas por adolescentes brasileiros: Pesquisa Nacional de Saúde do Escolar 2009, 2012 e 2015. Rev Bras Epidemiol 2018; 21(Suol. 1):e180014. 
11. Yang L, Zhang Y, Bovet P. Physical Fighting and Associated Factors among Adolescents Aged 13-15 Years in Six Western Pacific Countries. Int J Environ Res Public Health 2017; 14(11):1427.

12. Caledonia, KL, Wilson ML, El Grammal HA, Hagras AM. Physical fighting among Egyptian adolescents: social and demographic correlates among a nationally representative sample. Peer J 2013; 1:e125.

13. Instituto Brasileiro de Geografia e Estatística (IBGE). Pesquisa nacional de saúde escolar. Rio de Janeiro: IBGE; 2016.

14. World Health Organization (WHO). World Health Organization Noncommunicable diseases: country profiles 2011. Geneva: WHO Press; 2011.

15. Bala MO, Chehab MA, Al-Dashan A, Saadeh S, Al Khenji A. Violence among Adolescents in Qatar: Results from the Global School-based Student Health Survey, 2011. Cureus 2018; 10(7):e2913.

16. Senanayake SJ, Gunawardena S, Wickramasinghe $S$, Wickramasinghe C, Gunawardena NS, Lokubalasooriya $A$, Peiris R, Agarval N, Rani M. Prevalence and Correlates of Interpersonal Violence Among In-School Adolescents in Sri Lanka: Results From the 2016 Sri Lankan Global School-Based Health Survey. Asia Pac J Public Health 2019; 31(2):147-156.

17. Brook JS, Brook DW, Whiteman M. Growing up in a violent society: longitudinal predictors of violence in Colombian adolescents. Am J Community Psychol 2007; 40(1-2):82-95.

18. Nobre CS, Vieira LJES, Noronha CV, Frota MAN. Fatores associados à violência interpessoal entre crianças de escolas públicas de Fortaleza, Ceará, Brasil. Cien Saude Colet 2018; 23(12):4299-4309.

19. Assis SG, Avanci JQ, Santos NC, Malaquias JV, Oliveira RVC. Violência e representação social na adolescência no Brasil. Rev Panam Salud Publica 2004; 16(1):43-51.

20. Duke NN, Pettingell SL, McMorris BJ, Borowsky IW. Adolescent violence perpetration: Associations with multiple types of adverse childhood experiences. $\mathrm{Pe}$ diatrics 2010; 125(4):e778-e786.

21. Malta DC, Souza ER, Silva MMA, Silva CS, Andreazzi MAR, Crespo C, Mascarenhas MDM, Porto DL, Figueroa ALG, Morais Neto OL, Penna GO. Vivência de violência entre escolares brasileiros: resultados da Pesquisa Nacional de Saúde do Escolar (PeNSE). Cien Saude Colet 2010; 15(Supl. 2):3053-3063.

22. Borofsky LA, Kellerman I, Baucom B, Oliver PH, Margolin G. Community violence exposure and adolescents school engagement and academic achievement over time. Psychol Violence 2013; 3(4):381-395.
23. Lim SS, Vos T, Flaxman AD, Danaei G, Shibuya K, Adair-Rohani H, Amann M, Anderson HR, Andrews KG, Aryee M, Atkinson C, Bacchus LJ, Bahalim AN, Balakrishnan K, Balmes J, Barker-Collo S, Baxter A, Bell ML, Blore JD, Blyth F, Bonner C, Borges G, Bourne R, Boussinesq M, Brauer M, Brooks P, Bruce NG, Brunekreef B, Bryan-Hancock C, Bucello C, Buchbinder R, Bull F, Burnett RT, Byers TE, Calabria B, Carapetis J, Carnahan E, Chafe Z, Charlson F, Chen $\mathrm{H}$, Chen JS, Cheng AT-A, Child JC, Cohen A, Colson KE, Cowie BC, Darby S, Darling S, Davis A, Degenhardt L, Dentener F, Des Jarlais DC, Devries K, Dherani M, Ding EL, Dorsey ER, Driscoll T, Edmond K, Ali SE, Engell RE, Erwin PJ, Fahimi S, Falder G, Farzadfar F, Ferrari A, Finucane MM, Flaxman S, Fowkes FGR, Freedman G, Freeman MK, Gakidou E, Ghosh S, Giovannucci E, Gmel G, Graham K, Grainger R, Grant B, Gunnell D, Gutierrez HR, Hall W, Hoek HW, Hogan A, Hosgood 3rd HD, Hoy D, Hu H, Hubbell BJ, Hutchings SJ, Ibeanusi SE, Jacklyn GL, Jasrasaria R, Jonas JB, Kan H, Kanis JA, Kassebaum N, Kawakami N, Khang Y-H, Khatibzadeh S, Khoo J-P, Kok C, Laden F, Lalloo R, Lan Q, Lathlean T, Leasher JL, Leigh J, Li Y, Lin JK, Lipshultz SE, London S, Lozano R, Lu Y, Mak J, Malekzadeh R, Mallinger L, Marcenes W, March L, Marks R, Martin R, McGale P, McGrath J, Mehta S, Mensah GA, Merriman TR, Micha R, Michaud C, Mishra V, Hanafiah KM, Mokdad AA, Morawska L, Mozaffarian D, Murphy T, Naghavi M, Neal B, Nelson PK, Nolla JM, Norman R, Olives C, Omer SB, Orchard J, Osborne R, Ostro B, Page A, Pandey KD, Parry CDH, Passmore E, Patra J, Pearce N, Pelizzari PM, Petzold M, Phillips MR, Pope D, Pope 3rd CA, Powles J, Rao M, Razavi H, Rehfuess EA, Rehm JT, Ritz B, Rivara $\mathrm{FP}$, Roberts $\mathrm{T}$, Robinson $\mathrm{C}$, Rodriguez-Portales JA, Romieu I, Room R, Rosenfeld LC, Roy A, Rushton L, Salomon JA, Sampson U, Sanchez-Riera L, Sanman E, Sapkota A, Seedat S, Shi P, Shield K, Shivakoti R, Singh GM, Sleet DA, Smith E, Smith KR, Stapelberg NJC, Steenland K, Stöckl H, Stovner LJ, Straif K, Straney L, Thurston GD, Tran JH, Van Dingenen R, van Donkelaar A, Veerman JL, Vijayakumar L, Weintraub R, Weissman MM, White RA, Whiteford H, Wiersma ST, Wilkinson JD, Williams HC, Williams W, Wilson N, Woolf AD, Yip P, Zielinski JM, Lopez AD, Murray CJL, Ezzati M, AlMazroa MA, Memish ZA. A comparative risk assessment of burden of disease and injury attributable to 67 risk factors and risk factor clusters in 21 regions, 1990-2010: a systematic analysis for the Global Burden of Disease Study 2010. Lancet 2012; 380(9859):2224-2260.

24. Mercado-Crespo MC, Mbah AK. Race and ethnicity, substance use, and physical aggression among U.S. high school students. J Interpers Violence 2013; 28(7):1367-1384.

25. Chaveepojnkamjorn W, Pichainarong N. Current drinking and health-risk behaviors among male high school students in Central Thailand. BMC Public Health 2011; 11:233.

26. Abdalla RR, Massaro L, Queiroz CMA, Laranjeira R, Caetano R, Madruga CS. Association between drug use and urban violence: Data from the II Brazilian National Alcohol and Drugs Survey (BNADS). Addictive Behaviors Reports 2018; 7:8-13. 
27. Carvalho AP, Silva TC, Valença PAM, Ferreira SCFB, Colares V, Menezes VA. Consumo de álcool e violência física entre adolescentes: quem é o preditor? Cien Saude Colet 2017; 22(12):4013-4020.

28. Pardini DA, Loeber R, Farrington DP, Stouthamer -Loeber M. Direct protective factors for nonviolence. Am J Prev Med 2012; 43(2 Supl. 1):S28-S40.

29. Caledonia KL, Wilson ML, El Gralmal HA, Hagrass AM. Physical fighting among Egyptian adolescents: social and demographic correlates among a nationally representative sample. Peer J 2013; 1:e125.

30. Albuhairan F, Abou O, Sayed DA, Badri M, Alshari $\mathrm{S}$, Vries $\mathrm{N}$. The relationship of bullying and physical violence to mental health and academic performance: A cross-sectional study among adolescents in Kingdom of Saudi Arabia. Int J Pediatr Adolesc Med 2017; 4(2):61-65.

31. Malta DC, Machado ÍE, Felisbino-Mendes MS, Prado RR, Pinto MAS, Oliveira-Campos M, Souza MFM, Assunção AÁ. Uso de substâncias psicoativas em adolescentes brasileiros e fatores associados: Pesquisa Nacional de Saúde dos Escolares, 2015. Rev Bras Epidemiol 2018; 21(Supl. 1):e180004.

32. Peres CA, Rutherford G, Borges G, Galano E, Hudes ES, Hearst N. Family structure and adolescent sexual behavior in a poor area of Sao Paulo, Brazil. J Adolesc Health 2008; 42(2):177-183.

33. Elsaesser C, Gorman-Smith D, Henry D, Schoeny M. The Longitudinal Relation Between Community Violence Exposure and Academic Engagement During Adolescence: Exploring Families' Protective Role. J Interpers Violence 2020; 35(17-18):3264-3285.

34. Kipping RR, Smith M, Heron J, Hickman M, Campbell R. Multiple risk behavior in adolescence and socio-economic status: findings from a UK birth cohort. Eur J Public Health 2014; 2(1):44-49.

35. Shakeel MD, De Angelis CA. Can private schools improve school climate? Evidence from national representative sample. J School Choice 2018; 12(3):426-445.
36. Cotrim BC, Carvalho CG, Gouveia N. Comportamentos de saúde entre jovens estudantes das redes pública e privada da área metropolitana do Estado de São Paulo. Rev Saúde Pública 2000; 34(6):636-645.

37. Andrade FH. Can the school context moderate the protective effect of parental support on adolescents' alcohol trajectories in urban Chicago? Drug Alcohol Depend 2013; 133(2):330-337.

38. Instituto Nacional de Estudos e Pesquisas Educacionais Anísio Teixeira (INEP). Sinopse Estatística da Educação Básica 2015. Brasília: INEP; 2016.

39. Brito ALS, Hardmam CM, Barros MV. Prevalence and factors associated with the co-occurrence of health risk behaviors in adolescents. Rev Paul Pediatr 2015; 33(4):423-430.

40. Pierobon M, Barakb M, Hazratib S, Jacobsen KH. Alcohol consumption and violence among Argentine adolescents. J Pediatr (Rio J) 2013; 89(1):100-107.

Artigo apresentado em 22/11/2019

Aprovado em 01/06/2020

Versão final apresentada em 03/06/2020

Editores chefes: Romeu Gomes, Antônio Augusto Moura da Silva 